\title{
On the Two SAOR Iterative Formats for Solving Linear Complementarity Problems
}

\author{
H.Saberi Najafi \\ Department of Applied Mathematics, Islamic Azad university of Lahijan, Iran \\ Email:hnajafi@gulan.ac.ir \\ S.A.Edalatpanah \\ Department of Applied Mathematics, Islamic Azad university of Lahijan, Iran \\ Email: saedalatpanah@gmail.com
}

\begin{abstract}
Han et.al have applied two SAOR splitting formats for solving the linear complementarity problem. We improve them by introducing a class of preconditioners based on the SAOR methods. The convergences of the modified methods have been analyzed. We also show the applicability of the methods by numerical example.
\end{abstract}

Index Terms-preconditioning, SAOR methods, linear complementarity problem, convergence, $\mathrm{H}$-matrix

\section{Introduction}

The linear complementarity problem Abbreviated as $\operatorname{LCP}(M, q)$, is to find a vector $z \in R^{n}$ such that

$$
\left\{\begin{array}{l}
w=M z+q, \\
z \geq 0, w \geq 0, \\
z^{T} w=0,
\end{array}\right.
$$

where, $z^{T}$ denotes the transpose of the vector $z$.

This problem is fundamental decision and optimization problem. It arises in many scientific applications like economic equilibrium analysis, fluid flow analysis, game theory, and mathematical programming.

For more details (see [1-3] and the references therein). This problem has been intensely studied since 1960s and many methods for solving the $\operatorname{LCP}(M, q)$ have been introduced. Most of these methods originate from those for the system of the linear equations where may be classified into two principal classes, i.e. direct and iterative methods (see [1-3]).

Various authors recently have suggested different models in the frame of the iterative methods for the above mentioned problem.

For example, Yuan and Song in [4], based on the models in [5] proposed a class of modified AOR (MAOR) methods to solve $\operatorname{LCP}(M, q)$. Also when the system matrix $M$ is an $H$-matrix they proposed some sufficient conditions for convergence of the MAOR and

\footnotetext{
Corresponding author: S.A.Edalatpanah
}

MSOR methods.

Under certain conditions, Li and Dai in [6] and Han, Yuan and Jiang in [7] also studied GAOR and SAOR for solving $L C P(M, q)$ based on [4], respectively (to see that other iterative methods for $L C P(M, q)$ see [4-7] and the references therein).

In this paper, we will propose a modification of SAOR methods for $\operatorname{LCP}(M, q)$. To accomplish this purpose, SAOR methods are coupled with the preconditioning strategy. We also show that our methods for solving LCP are superior to the basic SAOR methods. Numerical experiment shows that the new methods are feasible and efficient for solving large sparse linear complementarity problems.

\section{Prerequisite}

We begin with some basic notation and preliminary results which will be refereed later.

Definition 2.1 [8-10].

(a) The matrix $\mathrm{A}=\left[\mathrm{a}_{\mathrm{ij}}\right]$ is nonnegative (positive) if $a_{i j} \geq 0\left(a_{i j}>0\right)$. In this case we write $A \geq 0(A>0)$. Similarly, for $\mathrm{n}$-dimensional vectors $\mathbf{x}$, which are $n \times 1$, matrices,

We can also define $\mathrm{x} \geq 0(\mathrm{x}>0)$.

(b) A matrix $\mathrm{A}=\left(a_{i j}\right)_{n \times n}$ is called a Z-matrix if for any $i \neq j, a_{i j} \leq 0$

(c) A Z-matrix is M-matrix, if $\mathrm{A}$ is nonsingular, and if $A^{-1} \geq 0$.

(d) A square matrix $\mathrm{A}=\left(a_{i j}\right)_{n \times n}$ is called M-matrix if $A=\alpha I-B ; B \geq 0$ and $\alpha>\rho(B)$; (we denote the spectral radius of $B$ by $\rho(B))$.

(e) For any matrix $\mathrm{A}=\left(a_{i j}\right)_{n \times n}$ the comparison matrix $\langle A\rangle=\left(m_{i j}\right) \in R^{n \times n} \quad$ is defined by:

$$
m_{i i}=\left|a_{i i}\right|, \quad m_{i j}=-\left|a_{i j}\right|, \quad i \neq j \quad 1 \leq i, j \leq n
$$


(f) The Matrix $\mathrm{A}=\left(a_{i j}\right)_{n \times n}$ is an H-matrix if and only if $\langle A\rangle$ is M-matrix.

Definition 2.2 [4-7] For $x \in R^{n}$, vector $\mathrm{x}_{+}$is defined such that $\left(\mathrm{x}_{+}\right)_{\mathrm{j}}=\max \left\{0, \mathrm{x}_{\mathrm{j}}\right\}, \mathrm{j}=1,2, \ldots, \mathrm{n}$. Then, for any $x, y \in R^{n}$, the following facts hold:
1. $(\mathrm{x}+\mathrm{y})_{+} \leq \mathrm{x}_{+}+\mathrm{y}_{+}$
2. $\mathrm{x}_{+}-\mathrm{y}_{+} \leq(\mathrm{x}-\mathrm{y})_{+}$
3. $|x|=\mathrm{x}_{+}+(-\mathrm{x})_{+}$
4. $\mathrm{x} \leq \mathrm{y}$ implies $\mathrm{x}_{+} \leq \mathrm{y}_{+}$.

Definition 2.3 [8-9] Let $A$ be a real matrix. The splitting $A=M-N$ is ,

(a) convergent if $\rho\left(M^{-1} N\right)<1$

(b) regular if $M^{-1} \geq 0$ and $N \geq 0$

(c) weak regular if $M^{-1} \geq 0$ and $M^{-1} N \geq 0$

(d) $M$-splitting if $M$ is $M$-matrix and $N \geq 0$.

Clearly an M-splitting is regular and a regular splitting is weak regular.

Lemma 2. $1[8,10]$ Let A be a Z-matrix. Then $\mathrm{A}$ is $M$ matrix if and only if there is a positive vector $\mathrm{x}$ such that $\mathrm{Ax}>0$.

Lemma 2.2 $[8,10]$ Let A $=\mathrm{M}-\mathrm{N}$ be an $M$-splitting of A. Then $\rho\left(M^{-1} N\right)<1$ if and only if $\mathrm{A}$ is $M$-matrix.

Lemma 2.3[9] Let A, B are Z-matrix and A is an $M$ matrix, if $\mathrm{A} \leq \mathrm{B}$ then $\mathrm{B}$ is also an M-matrix.

Lemma 2.4 [9] If $A \geq 0$, then

(1) A has a nonnegative real eigenvalue equal to its spectral radius,

(2) An eigenvector $\mathrm{x} \geq 0$, corresponds to $\rho(A)>0$

(3) $\rho(A)$ does not decrease when any entry of A is increased.

Lemma 2.5 [10] Let $\mathrm{T} \geq 0$. If there exist $\mathrm{x}>0$ and $\mathrm{a}$ scalar $\alpha>0$ such that

i) $\quad T x \leq \alpha x$, then $\rho(T) \leq \alpha$. Moreover, if

$$
T x<\alpha x \text {, then } \rho(T)<\alpha \text {. }
$$

ii) $T x \geq \alpha x$, then $\rho(T) \geq \alpha$. Moreover, if

$$
T x>\alpha x \text {, then } \rho(T)>\alpha \text {. }
$$

Lemma 2.6[4] $L C P(M, q)$ can be equivalently transformed to a fixed-point system of equations

$$
z=(z-\alpha E(M z+q))_{+} .
$$

Where $\alpha$ is positive constant and $E$ is a diagonal matrix with positive diagonal elements.

Lemma 2.7 [4-7] Let $M \in R^{n \times n}$ be an H-matrix with positive diagonal elements. Then the $\operatorname{LCP}(M, q)$ has a unique solution $z^{*} \in R^{n}$.
Let the matrix $M$ is split as

$$
\mathrm{M}=\mathrm{D}-\mathrm{L}-\mathrm{U} \text {, }
$$

where $D$ diagonal, $L$ and $U$ are strictly lower and upper triangular matrices of $M$, respectively. Then by choice of $\alpha E=D^{-1}$ and Lemma 2.6 we have

$$
z=\left(z-D^{-1}(M z+q)\right)_{+} .
$$

So, in order to solve $\operatorname{LCP}(M, q)$, SAOR iterative methods are defined in [7] as follows

$$
\begin{gathered}
z^{k+1}=\left(z^{k}-D^{-1}\left[-\gamma L z^{k+1}+(w(2-w) M+\gamma L) z^{k}+\right.\right. \\
w(2-w) q])_{+} .
\end{gathered}
$$

Also they proposed following model

$$
\begin{gathered}
z^{k+1}=\left(z^{k}-D^{-1}\left[-\gamma U z^{k+1}+(w(2-w) M+\gamma U) z^{k}\right.\right. \\
+w(2-w) q])_{+} .
\end{gathered}
$$

Where $w, \gamma \in R^{+}$.

The operator $f: R^{n} \longrightarrow R^{n}$, is defined such that $f(z)=\xi$, where $\xi$ is the fixed point of the system

$$
\begin{gathered}
\xi=\left(z-D^{-1}[-\gamma L \xi+(w(2-w) M+\gamma L) z\right. \\
+w(2-w) q])_{+} .
\end{gathered}
$$

Let

$$
\left\{\begin{array}{l}
Q=I-\gamma D^{-1}|L|, \\
R=\left|I-D^{-1}(w(2-w) M+\gamma L)\right| .
\end{array} .\right.
$$

Then in next lemma we have the convergence theorem, proposed in [7] for the SAOR methods.

Lemma 2.8 [7] Let $M \in R^{n \times n}$ be an H-matrix with positive diagonal elements and $0<\gamma \leq w \leq 1$. Then, for any initial vector $z^{0} \in R^{n}$, the iterative sequence $\left\{z^{k}\right\}$ generated by the SAOR methods $(2.4),(2.5)$ converges to the unique solution $\mathrm{z}^{*}$ of the $\operatorname{LCP}(M, q)$ and $\rho\left(Q^{-1} R\right)<1$.

\section{Preconditioned SAOR for $\operatorname{LCP}(M, q)$}

Preconditioning methods are the most authoritative techniques to improve the properties of the basic iterative methods. The main aim of preconditioning methods is to substitute the original matrix $M$ with an equivalent one i.e, $M^{\operatorname{Pr} e c}$, which has better properties concerning the computation of a solution (generally by a certain iterative methods). The two matrices are equivalent in the sense that, they have the same solution. Simple preconditioners of this type are the left matrix preconditioners. The left preconditioned is a nonsingular 
matrix $P$ and the preconditioned matrix is defined by $M^{\operatorname{Pr} e c}=P M$ where $P \approx M^{-1}$.

In this section, SAOR methods for LCP and the effect of preconditioning for these methods are coupled. In the literature, various authors have suggested different models of $(I+S)$-type preconditioner for linear systems $A=I-L-U$; where $I$ is the identity matrix and $L, U$ are strictly lower and strictly upper triangular matrices of $A$, respectively (see [11-15] and the references therein). These preconditioners have reasonable effectiveness and low construction cost. For example In 1987 Milaszewicz [11] presented the preconditioner of (I+S)-type, where the elements of the first column below the diagonal of A eliminate. Usui et al. In [12] considered the alternative preconditioner,with the following form

$$
\widehat{P}=I+L
$$

Generally, we want transform $M$ to $(I+S) M$. So let $M$ in (2.2) is nonsingular. Then preconditioning in $M$ is;

$$
\begin{aligned}
\bar{M}= & (I+S) M= \\
& D-L-U+S D-S L-S U \\
= & \bar{D}-\bar{L}-\bar{U} .
\end{aligned}
$$

Where $\bar{D}, \bar{L}, \bar{U}$ are diagonal, strictly lower and strictly upper triangular parts of $\bar{M}$, respectively .

And

$$
\bar{q}=(I+S) q
$$

We consider Usui et al's preconditioner as $(I+S)$. Therefore we have

$$
S=\left[\begin{array}{ccccc}
0 & 0 & \cdots & \cdots & 0 \\
-\frac{m_{21}}{m_{11}} & 0 & 0 & \cdots & 0 \\
-\frac{m_{31}}{m_{11}} & -\frac{m_{32}}{m_{22}} & 0 & \ddots & \vdots \\
\vdots & \vdots & \cdots & 0 & 0 \\
-\frac{m_{n 1}}{m_{11}} & -\frac{m_{n 2}}{m_{22}} & \cdots & -\frac{m_{n, n-1}}{m_{n-1, n-1}} & 0
\end{array}\right] .
$$

Note. We can also consider other (I+S)-type preconditioners but here, we use Usui et al's preconditioner, since convergence rate via using this preconditioner slightly is better than others; (see[13]).

Thus the preconditioned SAOR methods for LCP are:

\section{Format I:}

$z^{k+1}=\left(z^{k}-\bar{D}^{-1}\left[-\gamma \bar{L} z^{k+1}+(w(2-w) \bar{M}+\gamma \bar{L}) z^{k}\right.\right.$

$+w(2-w) \bar{q}])_{+}$,

\section{Format II:}

$$
\begin{aligned}
& z^{k+1}=\left(z^{k}-\bar{D}^{-1}\left[-\gamma \bar{U} z^{k+1}+(w(2-w) \bar{M}+\gamma \bar{U}) z^{k}\right.\right. \\
& +w(2-w) \bar{q}])_{+} .
\end{aligned}
$$

Lemma 3.1. Let $M$ be an H-matrix. Then the preconditioned $\bar{M}=(I+S) M$ also is H-matrix.

Proof. Let $M$ be an $H$-matrix then $<\mathrm{M}>$ is $M$-matrix and by Lemma 2.1

$$
\exists x>0 \text { S.T }<M>x>0 \text {. }
$$

Since $<\bar{M}>=(I+|S|)<M>$,

then

$$
<\bar{M}>x=(I+|S|)<M>x>0 .
$$

Therefore $\langle\bar{M}\rangle$ is M-matrix and the proof is completed.

Theorem 3.2. Let $M \in R^{n \times n}$ with positive diagonal elements be an H-matrix and $\bar{M}=(I+S) M$ is preconditioned form of $\mathrm{M}$ with preconditioner (3.3).

Then if

$$
\left\{\begin{array}{l}
\bar{Q}=I-\gamma \bar{D}^{-1}|\bar{L}|, \\
\bar{R}=\left|I-\bar{D}^{-1}(w(2-w) \bar{M}+\gamma \bar{L})\right|,
\end{array}\right.
$$

we have

$$
\rho\left(\bar{Q}^{-1} \bar{R}\right) \leq \rho\left(Q^{-1} R\right)<1 .
$$

Proof. By Lemma $3.1 \quad \bar{M}$ is an H-matrix. Hence $<\bar{M}>=\bar{Q}-\bar{R}$ is M-matrix and by Lemma 2.2 $\rho\left(\bar{Q}^{-1} \widetilde{R}\right)<1$. Since $(<\bar{M}>)<\bar{Q}$ by Lemma $2.3 \bar{Q}$ is $M$-matrix. Similarly $Q$ is also $M$-matrix .

Thus

$$
\left\{\begin{array}{l}
\bar{Q}^{-1} \geq 0, \bar{Q}^{-1} \bar{R} \geq 0 \\
Q^{-1} \geq 0, Q^{-1} R \geq 0
\end{array}\right.
$$

then by Lemma 2.4 (Perron-Frobenius Theorem) there exist a positive vector $\mathbf{x}$ such that

$$
\left(Q^{-1} R\right) x=\rho\left(Q^{-1} R\right) x .
$$

Therefore

$$
\begin{aligned}
& (Q-R) x=<M>x= \\
& Q\left(I-Q^{-1} R\right) x= \\
& \frac{1-\rho\left(Q^{-1} R\right)}{\rho\left(Q^{-1} R\right)} R x \geq 0 .
\end{aligned}
$$

Also for preconditioner(3.3) we have:

$$
\begin{aligned}
<\bar{M}>= & (I+|S|)<M>= \\
& (I+|S|)(D-|L|-|U|)= \\
& D-|L|-|U|+\left|K_{i}\right| D- \\
& \underbrace{|S| L \mid}_{D_{1}+L_{1}+U_{1}}-|S||U|=(\bar{D}-|\bar{L}|-|\bar{U}|),
\end{aligned}
$$

therefore 


$$
\Rightarrow\left\{\begin{array}{l}
\bar{D}=D-D_{1} \leq D \\
|\bar{L}|=|| L\left|+L_{1}\right| \geq|L| \\
|\bar{U}|=|| U\left|+U_{1}+\right| S|| U|-| S|D|,
\end{array}\right.
$$

and thus, $\bar{Q} \leq Q$ and in view of the fact that both $\bar{Q}, Q$ are $M$-matrices we have

$$
\bar{Q}^{-1}\left(I+K_{i}\right) \geq \bar{Q}^{-1} \geq Q^{-1} \text {. }
$$

Therefore,

$$
\begin{aligned}
0 & \leq\left[\bar{Q}^{-1}\left(I+K_{i}\right)-Q^{-1}\right](Q-R) x \\
& =\left(I-\bar{Q}^{-1} \bar{R}\right) x-\left(I-Q^{-1} R\right) x \\
& =Q^{-1} R x-\bar{Q}^{-1} \bar{R} x=\rho\left(Q^{-1} R\right) x-\bar{Q}^{-1} \bar{R} x .
\end{aligned}
$$

And by Lemma 2.5 we have; $\rho\left(\bar{Q}^{-1} \bar{R}\right) \leq \rho\left(Q^{-1} R\right)$.

Therefore by Lemma 2.8 the proof is completed.

Now, following [4-7], we show that in LCP, the convergence rate of preconditioned SAOR methods are faster than of the SAOR methods.

Theorem3.3. Let $M \in R^{n \times n}$ with positive diagonal elements be an H-matrix, $0<\gamma \leq w \leq 1$. Also $\bar{M}$ is preconditioned form of $\mathrm{M}$ with preconditioner (3.3). Then convergence rate of preconditioned SAOR methods are faster than of the SAOR methods.

Proof. We only prove FormatI; FormatII can be similarly verified. Let, iterative sequence $\left\{\mathrm{z}^{\mathrm{i}}\right\}$ $\mathrm{i}=0,1, \ldots$,generated by (3.4). From the assumption that $M$ is an H-matrix, it follows, by Lemma $3.1 \bar{M}$ is an $H$ matrix and therefore by Lemma 2.7 , the vector sequence $\left\{\mathrm{z}^{\mathrm{i}}\right\}$ is uniquely defined and the $\operatorname{LCP}(M, q)$ has a unique solution $z^{\bullet} \in R^{n}$. Similar to (2.6),we define the operator $v: R^{n} \longrightarrow R^{n}$, such that $v(z)=\bar{\xi}$, where $\bar{\xi}$ is the fixed point of the following system

$$
\begin{aligned}
& \bar{\xi}=\left(z-\bar{D}^{-1}[-\gamma \bar{L} \bar{\xi}+(w(2-w) \bar{M}+\gamma \bar{L}) z\right. \\
& +w(2-w) \bar{q}])_{+} .
\end{aligned}
$$

Let

$$
\begin{aligned}
& \bar{\psi}=v(x)=\left(x-\bar{D}^{-1}[-\gamma \bar{L} \bar{\psi}+\right. \\
& (w(2-w) \bar{M}+\gamma \bar{L}) x+w(2-w) \bar{q}])_{+} .
\end{aligned}
$$

By subtracting (3.6) \& (3.7), we get

$$
\left\{\begin{array}{l}
\bar{\xi}-\bar{\psi} \leq \\
\left((z-x)-\bar{D}^{-1}[\gamma \bar{L}(\bar{\xi}-\bar{\psi})+\right. \\
(w(2-w) \bar{M}+\overline{\mathcal{L}})(z-x)])_{+} \\
\& \\
\bar{\psi}-\bar{\xi} \leq \\
\left((x-z)-\bar{D}^{-1}[\bar{\gamma}(\bar{\psi}-\bar{\xi})+\right. \\
(w(2-w) \bar{M}+\bar{L})(x-z)])_{+} .
\end{array} .\right.
$$

Therefore, by above relations we have,

$$
\begin{aligned}
|\bar{\xi}-\bar{\psi}| & =(\bar{\xi}-\bar{\psi})_{+}+(\bar{\psi}-\bar{\xi})_{+} \\
& \leq \bar{Q}^{-1} \bar{R}|z-x|
\end{aligned}
$$

Thus from the definition of the preconditioned SAOR methods and (3.8) we can write,

$$
\left|z^{k+1}-z^{*}\right|=\left|v\left(z^{k}\right)-v\left(z^{*}\right)\right| \leq \bar{Q}^{-1} \bar{R}\left|z^{k}-z^{*}\right|
$$

Hence, the iterative sequence $\left\{\mathrm{z}^{\mathrm{k}}\right\}, \mathrm{k}=0,1, \ldots$, converges to $z^{*}$ if $\rho\left(\bar{Q}^{-1} \bar{R}\right) \leq 1$ and since by Theorem $3.2, \rho\left(\bar{Q}^{-1} \bar{R}\right) \leq \rho\left(Q^{-1} R\right)$ we conclude that for solving LCP ,the preconditioned SAOR iterative methods are better than of the SAOR methods form point of view of the convergence speed. So the proof is completed.

\section{4- NUMERICAL RESULTS}

In this section, we give an example to illustrate the results obtained in the previous sections. This example has been computed by using MATLAB7.

Example4.1. Consider $\operatorname{LCP}(M, q)$ with following system $M \in R^{N \times N}$ and $q \in R^{N}$

$$
\begin{gathered}
M=G \otimes I \otimes I+I \otimes F \otimes I+I \otimes I \otimes F \in R^{N \times N} \\
q=\left(-1,1, \ldots,(-1)^{n^{3}}\right)^{T} \in R^{N} .
\end{gathered}
$$

Where $I \in R^{N \times N}$ and $\otimes$ denotes the Kronecker product. Also $\mathrm{G}$ and $\mathrm{F}$ are $n \times n$ tridiagonal matrices given by

$$
\begin{aligned}
& G=\text { tridiagonal }\left[-\left(\frac{2+2 h}{12}\right), 1,-\left(\frac{2-2 h}{12}\right)\right], \\
& F=\text { tridiagonal }\left[-\left(\frac{2+h}{12}\right), 0,-\left(\frac{2-h}{12}\right)\right], \\
& \& h=1 / n \quad ; N=n^{3} .
\end{aligned}
$$

Evidently, $M$ is an $H$-matrix with positive diagonal elements. Then $\operatorname{LCP}(M, q)$ has a unique solution. Then, we solved the $\mathrm{n}^{3} \times \mathrm{n}^{3} \quad H$-matrix yielded by the iterative methods, and Preconditioned forms.

The initial approximation of $z$ is $z^{0}=(1,1, \ldots, 1)^{T} \in R^{N}$ and as a stopping criterion we choose $\left\{\begin{array}{l}\left\|\min \left(M z^{k}+q, z^{k}\right)\right\|_{\infty} \leq 10^{-6} \\ \left\|\min \left(\bar{M} z^{k}+\bar{q}, z^{k}\right)\right\|_{\infty} \leq 10^{-6} .\end{array}\right.$

In Tables 1,2, with several values we report the CPU time (CPU) and number of iterations (Iter) for the 
corresponding SAOR and preconditioned SAOR (PSAOR) methods with different parameters.

The results show that the preconditioned SAOR methods (both formats) can quickly compute satisfactory approximations to the solutions of above problem and we can easily see that these preconditioned methods are more efficient than the basic SAOR methods. For example for $\mathbf{N}=\mathbf{1 0 0 0}, \mathbf{w}=\mathbf{0 . 2}$ and $\gamma=\mathbf{0 . 2}$, When we use the preconditioned methods, the iteration steps are almost one hundred times less than the basic iterative methods and for time-consuming we have,

preconditioned methods $\approx 0.56 *$ basic methods.

Therefore, the preconditioned SAOR methods are superior to the basic iterative methods.

\section{Conclusions}

In this paper, we have proposed the preconditioned SAOR methods for linear complementarity problem and analyzed the convergence of these methods under certain conditions. We have also shown that, how the iterative methods for LCP are affected if the system is preconditioned by our models.

\section{References}

[1] Murty KG. Linear Complementarity, Linear and Nonlinear Programming[M ]. Heldermann Verlag: Berlin, 1988.

[2 ] Bazaraa MS, Sherali HD, Shetty CM. Nonlinear programming, Theory and algorithms[M ]. Third edition. Hoboken, NJ: Wiley-Interscience, 2006.

[3] Cottle RW, Pang JS, Stone RE. The Linear Complementarity Problem[M ]. Academic Press: New York, 1992.

[4] Yuan D, Song YZ. Modified AOR methods for linear complementarity problem [J]. Appl. Math. Comput ,2003, 140:53-67.

[5] Bai ZZ, Evans DJ. Matrix multisplitting relaxation methods for linear complementarity Problems [J]. Int. J. Comput. Math ,1997,63:309-326.

[6] Li Y, Dai P, Generalized AOR methods for linear complementarity problem[J]. Appl. Math. Comput 2007,188:7-18.

[7] Han X, Yuan D, Jiang Sh. Two SAOR Iterative Formats for Solving Linear Complementarity Problems[J]. IJITCS 2011, 2, 38-45.
[8] Varga RS. Matrix Iterative Analysis[M ]. second ed., Berlin :Springer; 2000.

[9] Frommer A , Szyld DB. H-splitting and two-stage iterative methods[J]. Numer. Math1992, 63:345-356.

[10] Berman A, Plemmons RJ. Nonnegative Matrices in the Mathematical Sciences[M ]. Academic Press: New York,1979.

[11] Milaszewicz JP. Improving Jacobi and GaussSeidel iterations[J]. Linear Algebra Appl 1987, 93: 161-170.

[12] Usui M, Niki H, Kohno T. Adaptive Gauss Seidel method for linear systems[J]. Intern. J. Computer Math 1994, 51:119-125.

[13] Li J.,C. Li, W.The Optimal Preconditioner of Strictly Diagonally Dominant Z-matrix[J]. Acta Mathematicae Applicatae Sinica, English Series. (2008) DOI: $10.1007 / \mathrm{s} 10255-006-6148-5$

[14] SaberiNajafi H , Edalatpanah SA. Some Improvements In PMAOR Method For Solving Linear Systems [J]. J.Info. Comp.Sci ,2011, 6:15-22.

[15] Hirano H , Niki H. Application of a Preconditioning iterative method to the computation of fluid flow [J]. Numer. Funct. Anal.And Optimiz, 2001,22:405-417.

H. Saberi Najafi: Associate Professor, Department of Applied Mathematics , University of Guilan\& Islamic Azad University of Lahijan, interested in Numerical linear algebra, Numerical Analysis and Numerical Modeling of the Oceans.( http://staff.guilan.ac.ir/saberi/?lg=1)

S.A.Edalatpanah: M.Sc in Applied Mathematics,academic member of Islamic Azad University of Lahijan, interested fields: Numerical Linear Algebra and Computational Optimization. 
Table1.The number of iteratins and CPU time for SAORI and its preconditioned form.

\begin{tabular}{|c|c|c|c|c|c|c|}
\hline \multicolumn{3}{|c|}{ Method } & \multicolumn{2}{|c|}{ SAOR Method I } & \multicolumn{2}{|c|}{$\begin{array}{c}\text { PSAOR } \\
\text { Method I }\end{array}$} \\
\hline $\mathbf{N}$ & $\mathbf{w}$ & $\gamma$ & CPU & Iter & CPU & Iter \\
\hline \multirow[t]{6}{*}{125} & 0.2 & 0.02 & 0.048064 & 159 & 0.015872 & 105 \\
\hline & 0.2 & 0.2 & 0.022187 & 148 & 0.015116 & 99 \\
\hline & 0.7 & 0.3 & 0.007537 & 50 & 0.004871 & 32 \\
\hline & 0.7 & 0.6 & 0.006558 & 43 & 0.004261 & 28 \\
\hline & 1.0 & 0.7 & 0.004372 & 29 & 0.002876 & 19 \\
\hline & 1.0 & 1.0 & 0.003975 & 25 & 0.002463 & 16 \\
\hline \multirow[t]{6}{*}{512} & 0.2 & 0.02 & 0.628651 & 304 & 0.402948 & 196 \\
\hline & 0.2 & 0.2 & 0.572845 & 281 & 0.367801 & 182 \\
\hline & 0.7 & 0.3 & 0.207923 & 96 & 0.128718 & 62 \\
\hline & 0.7 & 0.6 & 0.172554 & 82 & 0.111610 & 53 \\
\hline & 1.0 & 0.7 & 0.113595 & 57 & 0.071354 & 36 \\
\hline & 1.0 & 1.0 & 0.094838 & 46 & 0.061351 & 29 \\
\hline \multirow[t]{6}{*}{1000} & 0.2 & 0.02 & 2.397730 & 428 & 1.535994 & 276 \\
\hline & 0.2 & 0.2 & 2.478125 & 395 & 1.398550 & 256 \\
\hline & 0.7 & 0.3 & 0.844936 & 137 & 0.538365 & 88 \\
\hline & 0.7 & 0.6 & 0.758608 & 116 & 0.520795 & 75 \\
\hline & 1.0 & 0.7 & 0.505762 & 81 & 0.351534 & 52 \\
\hline & 1.0 & 1.0 & 0.404629 & 65 & 0.228767 & 41 \\
\hline \multirow[t]{6}{*}{2191} & 0.2 & 0.02 & 18.183986 & 700 & 11.799977 & 456 \\
\hline & 0.2 & 0.2 & 16.727265 & 645 & 10.948800 & 422 \\
\hline & 0.7 & 0.3 & 6.045586 & 232 & 3.843100 & 149 \\
\hline & 0.7 & 0.6 & 5.078971 & 196 & 3.262716 & 126 \\
\hline & 1.0 & 0.7 & 3.553875 & 137 & 2.256743 & 87 \\
\hline & 1.0 & 1.0 & 2.829414 & 109 & 1.740109 & 67 \\
\hline
\end{tabular}

Table2.The number of iteratins and CPU time for SAORII and its preconditioned form.

\begin{tabular}{|c|c|c|c|c|c|c|}
\hline \multicolumn{3}{|c|}{ Method } & \multicolumn{2}{|c|}{$\begin{array}{l}\text { SAOR Method } \\
\text { II }\end{array}$} & \multicolumn{2}{|c|}{$\begin{array}{l}\text { PSAOR } \\
\text { Method II }\end{array}$} \\
\hline $\mathbf{N}$ & $\mathbf{w}$ & $\gamma$ & CPU & Iter & CPU & Iter \\
\hline \multirow[t]{6}{*}{125} & 0.2 & 0.02 & 0.023491 & 159 & 0.016078 & 105 \\
\hline & 0.2 & 0.2 & 0.023103 & 148 & 0.015001 & 98 \\
\hline & 0.7 & 0.3 & 0.007406 & 50 & 0.004754 & 32 \\
\hline & 0.7 & 0.6 & 0.006423 & 43 & 0.004287 & 28 \\
\hline & 1.0 & 0.7 & 0.004504 & 30 & 0.002922 & 19 \\
\hline & 1.0 & 1.0 & 0.003857 & 25 & 0.002374 & 16 \\
\hline \multirow[t]{6}{*}{512} & 0.2 & 0.02 & 0.629974 & 304 & 0.445050 & 195 \\
\hline & 0.2 & 0.2 & 0.578688 & 282 & 0.430417 & 181 \\
\hline & 0.7 & 0.3 & 0.199673 & 96 & 0.134616 & 61 \\
\hline & 0.7 & 0.6 & 0.164254 & 82 & 0.114408 & 52 \\
\hline & 1.0 & 0.7 & 0.116346 & 57 & $\mathbf{0 . 0 7 4 1 5 7}$ & 36 \\
\hline & 1.0 & 1.0 & 0.110489 & 47 & 0.070957 & 28 \\
\hline \multirow[t]{6}{*}{1000} & 0.2 & 0.02 & 2.365414 & 428 & 1.520182 & 276 \\
\hline & 0.2 & 0.2 & 2.226123 & 396 & 1.585317 & 254 \\
\hline & 0.7 & 0.3 & 0.754636 & 138 & 0.480856 & 88 \\
\hline & 0.7 & 0.6 & 0.644249 & 117 & 0.444339 & 74 \\
\hline & 1.0 & 0.7 & 0.446212 & 82 & 0.279290 & 51 \\
\hline & 1.0 & 1.0 & 0.377697 & 66 & 0.218460 & 40 \\
\hline \multirow[t]{6}{*}{2191} & 0.2 & 0.02 & 18.17974 & 700 & 11.874742 & 456 \\
\hline & 0.2 & 0.2 & 16.73903 & 646 & 10.893500 & 420 \\
\hline & 0.7 & 0.3 & 6.03269 & 232 & 3.846774 & 148 \\
\hline & 0.7 & 0.6 & 5.08732 & 197 & 3.205456 & 124 \\
\hline & 1.0 & 0.7 & 3.54065 & 137 & 2.342892 & 85 \\
\hline & 1.0 & 1.0 & 2.85332 & 3110 & 1.675702 & 65 \\
\hline
\end{tabular}

